\title{
5 Young students and future teachers as passengers on the Logo engine
}

\author{
Ivan Kalas and Andrej Blaho \\ Department of Informatics Education \\ Comenius University, Bratislava \\ Slovak Republic
}

\begin{abstract}
In 1994 we released a new version of the Logo language. We now concentrate on research into new educational possibilities offered by this environment. We want to provide Logo users with a strong and flexible developers' tool for exploring, creating and playing. Together with our university students - future teachers of mathematics and informatics - we run a seminar on implementing informatics in primary and lower secondary education. The goal of the seminar for the 1995/96 school year was to develop a modern one-year course on informatics for lower secondary students, supported by a series of open microworlds which should provide creative laboratories to explore major ideas embedded in turtle geometry, mathematics, multimedia, logic and algorithmics.
\end{abstract}

\section{Keywords}

Programming language Logo, curriculum development, open learning, teacher education, tools.

\section{INTRODUCTION}

The main thrust in teaching Logo is the process rather than the final outcome of an activity (Shimabukuro, 1988).

In 1994 we released a new version of the Logo language, (Blaho et al., 1994; Stuur, 1994), with the ambition to make use of all the powerful features of the Windows environment and to provide complete support for turtle geometry, enhanced Logo data structures, multimedia, multiple turtles and the handling of complex graphics. Since then our Logo has appeared in more than ten national 


\section{Information and communications technologies in school mathematics}

editions throughout several countries (titled SuperLogo in the UK, the Netherlands and others; Comenius Logo in Central Europe; Multilogo in Greece and MegaLogo in Portugal and Brazil). As the number of users of this environment is growing considerably in schools, in families, in teachers education, in educational institutions for handicapped children, etc., we are now concentrating our research on new educational possibilities offered by this environment. Our approach is to provide Logo users with a strong and flexible developers' tool for exploring, creating and playing - the users being either young people, students, teachers or educational software developers. We believe that in a well designed open computer environment, learning can take place through the active participation in the development and use of microworlds.

Our educational research is based on close co-operation with our university students-future teachers of informatics and mathematics. It is important and highly motivating to work with future teachers as they are both very enthusiastic and very critical young people. Several of them are already involved as assistant teachers in experimental informatics courses our department runs in lower secondary schools (Blaho et al., 1995)

\section{LOWER SECONDARY INFORMATICS EDUCATION}

Two years ago we decided to start a seminar with our university students on implementing informatics in primary and lower secondary education. Every year we work with a group of students on a specified goal. In 1996 the goal was to develop a one-year Logo-based course on informatics for lower secondary students (between 10 and 14 years old). We tried to specify the objectives of the course, its conception and approach, and to design (and if possible, also implement) a series of open microworlds to support the course. These microworlds should cover all the major topics and provide young students with computer laboratories to explore important ideas within turtle geometry, mathematics, multimedia, logical reasoning and algorithmics. We have decided to stress creativity, playfulness and exploratory work and have specified the general educational goals as follows:

- to build a comfortable feeling in students when working with information technologies;

- to develop creativity, independence and personality (through the creation of animated stories, for example);

- to develop problem solving skills, logical reasoning and algorithmic thinking;

- to support co-operative work in projects;

- to support a procedural approach in the exploration of mathematical concepts such as distance, angles, polygons as results of computational processes in the use of turtle geometry;

- to combine different media for expressing more complex phenomena. 
We decided to have the course Logo-based and Logo-centred, therefore we also specified the minimum turtle geometry and programming concepts which should be covered within the major topics of the course as follows:

- elementary single turtle commands;

- the concept of angle and polygon;

- the concept of repeating, simple branching by if, the concept of userdefined procedure, procedures with inputs, simple variables through procedure's parameter/parameters;

- the concept of stepping the computation (i.e. pausing the computation at each forward, back, left and right commands);

- introduction to multiple turtles;

- introduction to multimedia control.

In the following section we summarise the developments in 12 main topics of the course. We always specify the goals to be reached, concepts to be explored and microworlds to be used within the topic.

The first topic is completely devoted to exploring Logo demo projects for each of the other topics. In this way students will intuitively recognise basic concepts, ways of communication, the environment, etc. Some demo Logo projects will illustrate applications of IT for other subjects (mathematics, geography, arts, cross-curricular projects etc.).

\section{THE OUTLINE OF THE COURSE}

\section{Demo projects}

Students will become familiar with the environment of Windows and Logo by navigating through a collection of simple Logo projects (offered in the Demo Projects Window of Logo). Most of them are simple interactive games, in which students use the mouse to control one or several active objects in the graphics screen and thus solve simple problems. They are given the opportunity to discover the concept of a turtle (or many turtles) and the way it can be controlled: making it go forward, turn left and right, turning its pen up and down, changing colours, etc. From the informatics point of view, they will intuitively experience some fundamental concepts, e.g., data and data representation, as well as engage in problem solving strategies and processes.

Figure 1 illustrates the Turtle Writer demo project in which students work with multiple representations of data. In the upper left part of the screen there is a pile of letters (printed on small square cut-outs), in the upper right part there is a pile of small pictures (printed on the same size cut-outs). If a student clicks a letter or presses the corresponding key on the keyboard, the letter starts to move downwards and is correctly positioned in the line (or lines) constructed in the lower part of the screen. In this way the student types the story. Some words, 
however, do not need letters - they will be expressed by clicking a picture. The picture will move by itself and will stop at the end of the sentence. In this way, a combined text-pictures story is easily created to form a unified result (with its parts represented in different ways).
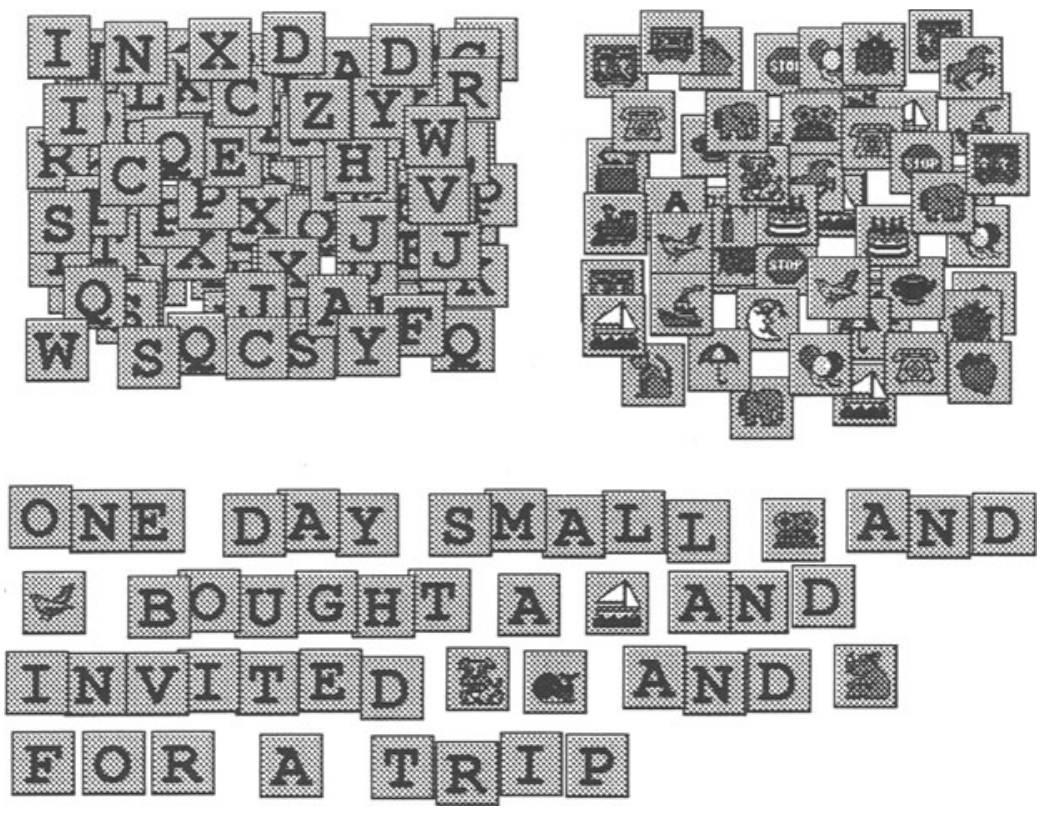

Figure 1 A story in words and pictures.

\section{Paint and Turtle Geometry}

We use Paint or Paintbrush bitmap editor as an introduction to Windows because it is simple, attractive, intuitive and it supports the idea of co-operation among Windows applications. If students create a drawing in Paint and paste it through the Clipboard into the Logo graphics screen as its background, several important informatics concepts are illustrated.

Such co-operation between Paint and Logo has inspired us to develop a microworld at the border between them: the graphical cursor is either in the Paint mode (with many well-known tools), or we turn it into the turtle mode. In such case, the graphical cursor (a pen) turns into a turtle which can be controlled in the usual Logo way (again, with many well-known tools). In fact, both modes are quite similar if we communicate with the cursor through direct manipulation. However, the turtle mode also makes it possible to control the cursor through Logo commands (and later even through Logo user-defined procedures, for 
example, if we define a simple house command, it can be run once or several times in different positions, pen colours and pen widths and then we can switch into the Paint mode, fill some parts of the houses and add smoke to each house in a traditional free-hand mode).

We will later use the same microworld to present a kind of Logo macro recorder to students: the complete sequence of Paint mode steps or turtle mode steps can be recorded any time and re-used later (an early introduction to the concept of a procedure). Working with such integrated environments will illustrate several important techniques as well as provide opportunities for printing and scanning.

\section{Playing Instruction Cards}

Shimabukuro (1988) introduced the idea of 'Surprise Programs'. These are working sheets of six to ten printed rectangles with simple Logo instructions such as fd 100, home, fd 10000, rt 45 (or even incomplete instructions of the form fd ?, etc.). The sheets are cut into little cards, shuffled and organised into a random, or surprise paper program. We have found this idea to be highly inspiring - a strong interactive laboratory for developing sequential thinking may be based on the concept of constructing and running sequences of similar instruction cards.

Students will find a collection of (complete) instruction cards on the screen. By choosing and dragging the cards into the other half of the screen, students will then construct a short sequence and be able to 'run' this. The concept of running a sequence of instructions will thus be developed. Later on, cards with incomplete instructions, like fd ?, setpc ?, setbg ? or rt ?, will be used as well. Students will replace unspecified inputs on the cards by specific values: if they click a particular symbol, palette or ruler or another chooser will open.

Such tools provide students with an interactive laboratory for experimenting with particular commands, different inputs (like $50,1000,-50,0$ or 1 for the forward command) and short sequences of instructions.

\section{Build Your Own Town Project (basic concepts in the Logo environment and language)}

This is a simple project with surprisingly deep didactics-reported in detail in Kalas and Blaho (1995). Students build a town by making the turtle (with penup) navigate through a simple background picture using basic turtle commands. Two additional commands are provided by the microworld, enabling the student to build a house and/or to plant a tree (house and tree)-if they are used, the turtle stamps an image of a randomly chosen house or tree at its current position. Students in this way explore several important features of the Logo environment and language. If they fail to type an input to fd or $r t$ or other primitive command, Ruler chooser or Compass chooser or Palette chooser is displayed instead of an error message. These interactive graphical tools help 
students specify inputs to commands. Other features and concepts to be explored in this demo project are more instructions in one line, Buttons Window, Memory Window, MegaPaint Image editor (to create new shapes and images) and elementary user-defined procedures.

All data including background bitmap and shapes of houses, trees, birds, etc., are directly accessible and can be modified or created by students directly within the environment. In this way, unexpected modifications of the project can be developed with nearly no effort, for example, turning 'Build Your Own Town' into 'Build Your Own Zoo'.

\section{Repeating}

Our experience has indicated that students are highly reluctant to replace linear sequence thinking by an iterative repeat structure. The argument that the instruction repeat 4 [fd 100 rt 90] is shorter and more transparent than four identical lines of a pair of instructions fd 100 rt 90 is not easily accepted. (The argument that repeat 4 [fd $100 \mathrm{rt} \mathrm{90]} \mathrm{introduces} \mathrm{a} \mathrm{new}$ and single abstract concept of a square is perfectly valid but would seem to be unsuitable for younger students.) Therefore our future teachers try to develop several microworlds, which will motivate the concept of iteration as a natural way of planning repeated actions.

If the goal is to build several streets (within the Build Your Own Town project) with dozens or hundreds houses and trees in regular rows, a kind of competition will make it obvious that these are real tasks which can be solved much quicker by the repeat structure than by a long linear sequence of instructions.

In another microworld, students are presented with a sequence of simple tasks to collect fruits from an apple tree (or from an alley of apple trees). However, the tasks are accompanied with stronger and stronger constraints so that the repeat solutions are better than linear ones. First, students will have the opportunity to read and interpret repeat instructions in certain templates, later they will fill in missing parts of the templates offered by the microworld, finally, they will be expected to construct such instructions themselves.

\section{Angle Laboratory}

Angles and negative numbers are probably the most complex mathematical concepts which might be developed through explorations within the Logo environment. The procedural approach is highly motivating here, for example, turtle's reactions to fd, bk, rt and it commands can be observed, undone or redone at any time. In previous activities, students have already worked with angles-most often with the interactive support of the Compass chooser-now, in several follow-up microworlds, they are given the opportunity to further develop the concept of angle and extend their planning skills. In the water slalom, students are to plan a zigzag trajectory of a sailing-boat so that it avoids all poles 
and obstacles and reaches the goal. Several correct solutions exist, some of them being better than others (if we suppose that all boats move at the same speed).

Beside such procedural microworlds, students are also to explore the concept of angle in several more traditional mathematics assignments. Figure 2 shows this implemented in an interactive Logo angle microworld. The students are to reconstruct the same angle as a given one, compare the two angles, add the two angles, double an angle, etc.

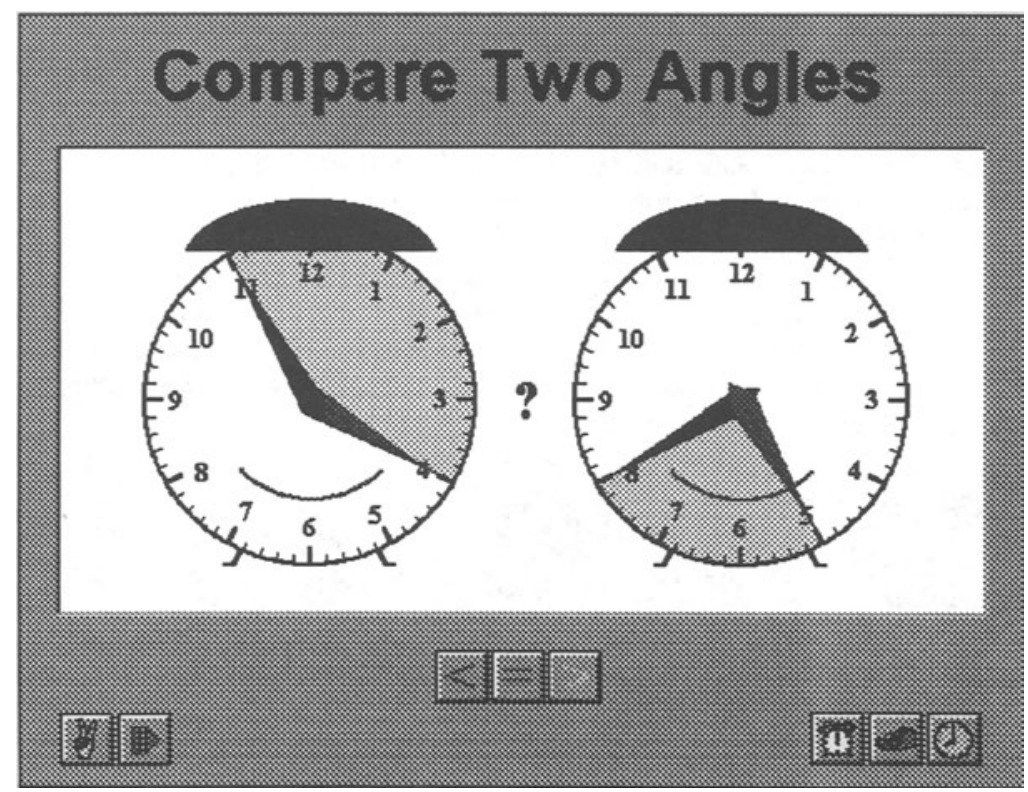

Figure 2 Angles on the clock face

\section{The World of Polygons}

Complex compositions can be created by combining polygons of different colours, sizes, filling patterns, number of nodes, etc. Regular polygons can be created by using the Logo tools (e.g., specifying the number of nodes, size, and colour - both through direct manipulations and through Logo language constructions), then dragged and placed in the working place.

Other microworlds provide a grid of dots environment in which students are able to create their own irregular polygons (first only with horizontal, vertical and 45 degree diagonal edges and later with other diagonal edges). In a tangram way, students are able to divide a rectangle into several irregular (triangular or rectangular) sub-parts. The parts may be dragged within the grid world and thus combined into manifold shapes. Later, each piece may be multiplied into as many 
copies as necessary. The goal is to cover the whole working area by fitting the pieces close together with no holes in-between.

Another kind of activity is that of exploring the area of each irregular polygon by counting the number of elementary squares covered by the polygon. Thus one is able to compare the areas of the two polygons.

\section{Multiple Turtles}

In so far as Logo makes it possible to create and control several active objectsturtles, students will have an opportunity to develop their own complex compositions in the graphics screen. These are likely to consist of imported background bitmaps (e.g. through the use of the Clipboard) from the Paint or similar editor, plus a collection of objects with mutually independent behaviour. Students are then able to use all the tools of the microworld to set shapes, directions, speed, etc., for each object.

Figure 3 is an example of the output (for a point in time) for which students constructed a suburban area of family houses and connecting roads. Multiple active objects - cars and planes-have been placed into the area where they move by themselves, without any additional control. The whole composition illustrates a living environment with multiple active agents.

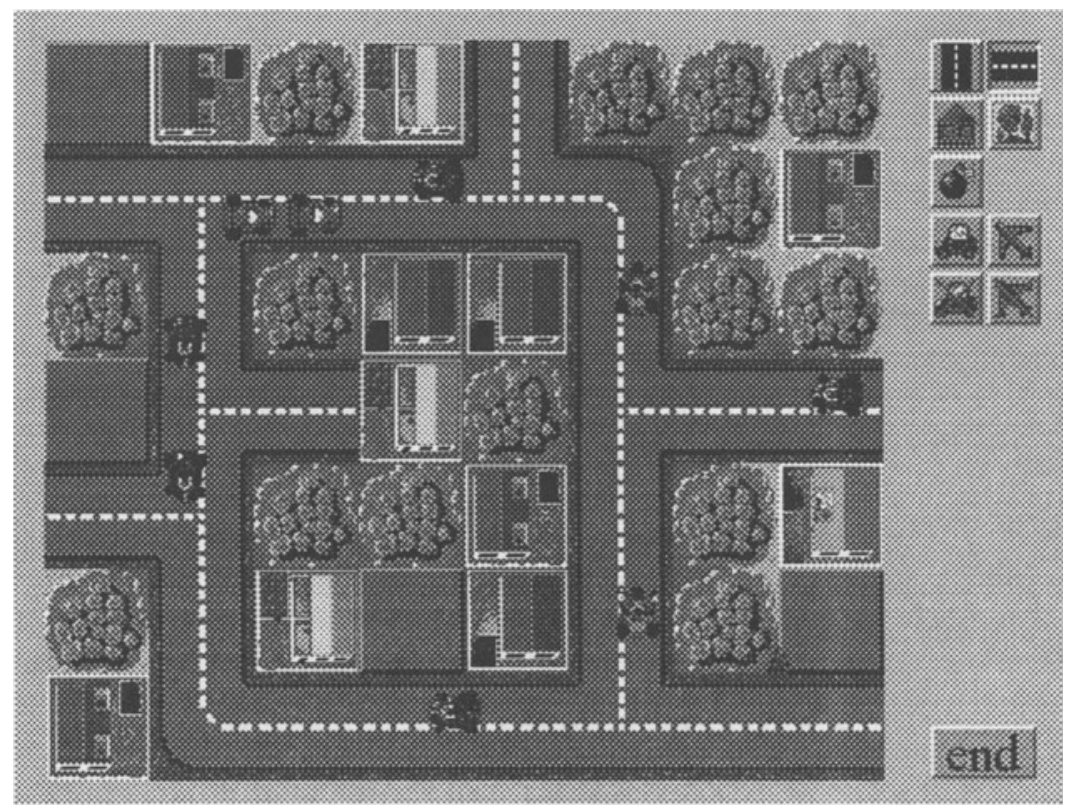

Figure 3 A suburban area-houses and roads.

In a more complex microworld, students are able to control all active objects by creating a kind of planning agenda. That is, they are able to specify time 
sequences of events to take place on the screen. So far, however, the objects cannot expose any alternative behaviour depending on, for example, their actual positions, the background colour behind them, or the presence of another object in their neighbourhood. The only control available to the student is simple independent timing planned for each object.

A further example of an activity (from a lower secondary school in Rio de Janeiro) is a project, in which students designed (out of many polygons) a scheme of a town with a piece of sea shore, or a lake or a river, with an airport and motor ways, populated by boats, planes and cars. Although the project is quite simple, some serious planning decisions had to be taken by the students-all active objects had to be created in several layers so that no plane was ever overlapped by a car or a boat, etc.

\section{Conditions and Events}

This topic is, from the conceptual point of view, the most complex of all since its goal is to develop some sense of intuitive alternative thinking - the third control structure the students will encounter in this course. While in the previous topic all objects were moved according to a time agenda, now students are advised to specify several events which should take place if certain conditions are met. The language of the microworld is very simple. A predefined set of possible conditions are offered to the user together with a set of possible reactions.

Students are to compose a kind of a board game with regular square grid, where each square can be coloured by selecting from a palette. The language of conditions consists of one-word tests (with no inputs) of the type: staying.on.red?, staying.on.blue?, etc. Students are to specify a reaction associated with each condition, for example: if staying.on.blue? [turn.right go 3 turn.left].

In this way, students are to create their own games with their own playing rules-by colouring some of the squares, by specifying the corresponding reactions and defining the goal to be reached.

\section{Working with Texts}

Using the work of Goldenberg and Feurzeig (1987) we have decided to include a topic with seemingly different data structures - natural language texts. However, even this text-oriented laboratory is to be implemented within the graphics screen. Each piece of text will be represented and processed as a structured sequence of certain graphics objects (corresponding to words). In this way, we expect to achieve high interactivity and visualisation of the 'laboratory'.

Students will be given the opportunity to interactively construct a general diagram (a template) of a class of similar sentences. At first, the template will represent very simple sentences, later it will represent compound sentences (of certain structures) or even the whole paragraphs. The templates will not consist of traditional grammatical categories (like subject and predicate), rather it will 
50 Information and communications technologies in school mathematics

consist of semantic categories (like somebody, something, a man, when, doing.what, with.whom, from.where, good.relation, bad.relation). Students will then specify sets of words which fit into each category and let the computer generate random instances (sentences and paragraphs) of their text templates.

The text generation microworld should support co-operative work and students will have a tool to combine their random sentences into random dialogues or stories.

\section{Animation Studio}

This microworld is a follow up on the Multiple Turtles laboratory. Objects of the previous activities were traditional turtles with certain shapes (used to express actual headings of the turtles - like the basic triangle shape, or shapes consisting of a single frame - a car, a plane, etc.). In the Animation Studio students are to populate their compositions with animation turtles with sequences of frames as their shapes. In SuperLogo, sequences of frames (called images) are used to express active movements of the characters-turtles. Images are new first-order data objects which can be set to turtles by the setshape command and can be easily processed with the Logo language (in a similar way to processing lists and words) in the (new) Logo environment. In the Animation Studio students will be able to develop and specify the shapes, movements and behaviour of turtle objects in the screen by developing and implementing their own scripts.

Figure 4 illustrates a sequence of frames created by a Dutch student to express the real movements of a ship in waves.

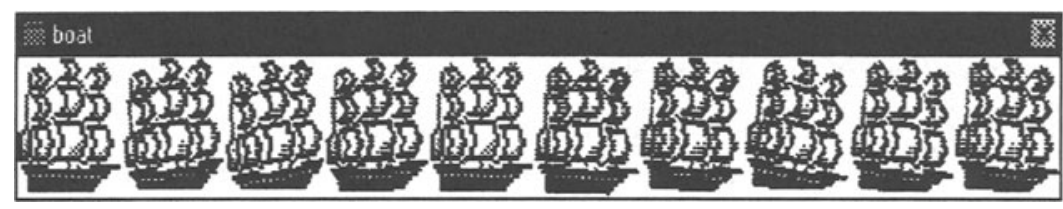

Figure 4 Movement of a ship in waves.

\section{Multimedia Studio}

We believe that students will appreciate the availability of tools which enable them to include sounds, melodies and video sequences into their animated compositions. These items will either be available from a library of ready made samples or recordings by students themselves in the corresponding Windows applications. Each particular event or tool of a student's composition can be supported by voice comments-recording them is always an amusement and running them gives a new dimension to their creations.

Students, working in such an open multimedia studio, will be able to engage in the process of developing scripts, creating backgrounds, characters (living objects) and their shapes, and choosing or developing sounds and melodies. 


\section{DISCUSSION}

We are aware of several shortcomings of the outcome of the 1996 seminar. Not all microworlds resulting from it are promising, not all of them have been realised, they are a kind of pilot implementations of ideas and discussions which have not yet been evaluated. In spite of that, we find this experience very important (both for us and for future teachers): in developing educational environments for students these first intuitive attempts have been important in that they have aided the clarification of new ideas as well as provided insights as to whether or not particular decisions have potential in other work.

\section{Acknowledgement}

This research has been partly supported by MATch, INCO Copernicus Project 960106.

\section{REFERENCES}

Blaho, A., Kalas, I. and Matusova, M. (1994). Environment for Environments: New Metaphor for Logo. In J. Wright and D. Benzie (eds.) Exploring a New Partnership: Children, Teachers and Technology, Amsterdam: IFIP, 153-166.

Blaho, A., Kalas, I. and Matusova, M. (1995). Experimental Curriculum of Informatics for 11 Year Old Children. In D. Tinsley and T. van Weert (eds.) World Conference on Computers in Education VI, IFIP, London: Chapman \& Hall, 829-841.

Goldenberg, E. and Feurzeig, W. (1987). Exploring Language with Logo. Boston: The MIT Press.

Kalas, I. and Blaho, A. (1995). Not All Birds Are Turtles: Developing Teaching/Learning Environments in Initial Teacher Training at Comenius University. In B. Collis, I. Nikolova and K. Martcheva (eds.), Information Technologies in Teacher Education, Paris: UNESCO, 222-235.

Shimabukuro, G. (1988). Thinking in Logo: A Sourcebook for Teachers of Primary Students, Addison-Wesley.

Stuur, A. (1994). Programmeren voor Kinderen met SuperLogo (in Dutch). Utrecht: A. W. Bruna Informatica. 


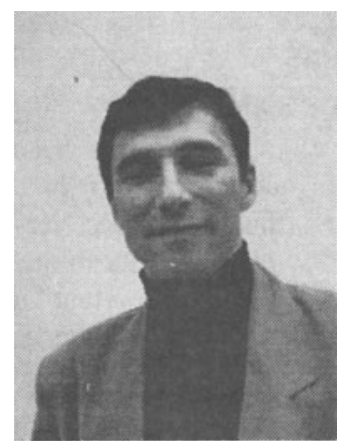

Ivan Kalas, associate professor at Comenius University, Bratislava, received his $\mathrm{PhD}$. in the field of knowledge representation, an area of informatics in which he has given lectures for ten years. In 1991 he published a book Another Programming: May I introduce LISP? on symbolic processing for upper secondary school students. $\mathrm{He}$ is interested in incorporating algorithmic thinking principles into basic and secondary education. With Andrej Blaho he co-authored educational $C D$ Thomas the Clown's Circus. His book of tales for children was translated into five foreign languages. He collaborated with Andrej Blaho and Peter Tomcsanyi in the production of Comenius Logo for Windows. He is the head of the Department of Informatics Education and is a representative of Slovak Republic in TC3, the Education Committee of IFIP.

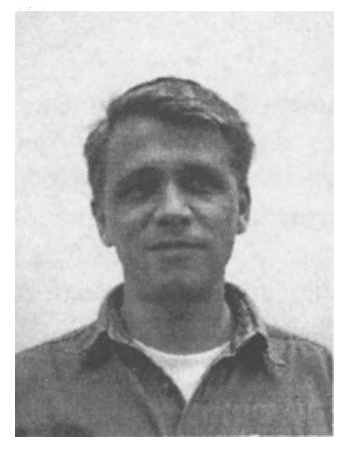

Andrej Blaho is a senior lecturer at Comenius University where he gives lectures on introductory and advanced programming. He has taken part in several major projects developing interactive educational environments for students. For five years he has published a series of articles on Logo and Karel the Robot in a teenage scientific magazine. He wrote the educational materials for teachers of programming and lab instructors. Andrej is the co-author of an activity book on problem solving, which received the Publisher's Award in 1991. He worked with Ivan Kalas and Peter Tomcsanyi on the development of Comenius Logo for Windows. 